\title{
Performance Analysis of a Secondary User in Cognitive Radio over Generalized-Gamma Fading Channels
}

\author{
Sultan Aldırmaz Çolak (iD \\ Department of Electronics and Communication Engineering, Kocaeli University, Kocaeli, Turkey
}

Cite this article as: Aldırmaz Çolak S. Performance Analysis of a Secondary User in Cognitive Radio over Generalized-Gamma Fading Channels. Electrica, 2019; 19(2): 166-172.

\section{ABSTRACT}

In this paper, the performance of a secondary user (SU) in an underlay cognitive radio (CR) network over a generalized-gamma (GG) fading channel in terms of three performance metrics, namely, outage probability, ergodic capacity, and bit error rate (BER), is investigated. According to the underlay approach, the transmit power of the SU should be limited to avoid interference to primary users (PUs). The performance metrics of the SU in the underlay CR network are theoretically derived and also obtained with Monte Carlo simulations for a GG fading channel with different fading parameters. To demonstrate the effect of limited power on the performance metrics, three different maximum transmit power levels $\left(P_{\max }\right)$, namely, 10, 20, and $30 \mathrm{~dB}$, are used. The simulation results lead to three conclusions: First, when high values are chosen for the fading parameters $(a, c)$, the fading channel gets less distorted and the performance metrics improve. The second conclusion is that the BER performance gets better as the limited power increases. The final conclusion is that the BER performance of SUs improves as the interference power that PUs can tolerate increases.

Keywords: Cognitive radio, generalized-gamma fading channel, underlay approach.

\section{Corresponding Author: \\ Sultan Aldırmaz Çolak}

E-mail:

sultan.aldirmaz@kocaeli.edu.tr

Received: 23.03 .2019

Accepted: 05.05.2019

C Copyright 2019 by Electrica Available online at

http://electrica.istanbul.edu.tr

DOI: 10.26650/electrica.2019.19017

Content of this journal is licensed under a Creative Commons Attribution-NonCommercial 4.0 International License.

\section{Introduction}

Cognitive radio (CR) was proposed by Mitola afforded an opportunity for unlicensed users to efficiently use the spectrum without any destructive effect on licensed users [1, 2]. A large number of authors have been studying the CR concept to cope with the spectrum scarcity problem due to the increasing demand for wireless communications and the use of new-generation communication systems [3,4]. There are three different approaches in CR: underlay, overlay, and interweave. In the underlay approach, while both licensed (primary) and unlicensed (secondary) users simultaneously transmit their signals, the transmission power of secondary users (SUs) is limited within a predefined threshold. Therefore, they do not have any disruptive effect on primary users (PUs). However, in the interweave approach, SUs need to seek an opportunity to exploit the spectrum when it is not being utilized by PUs. Finally, in the overlay approach, PUs and SUs behave as partners and PUs share their transmission parameters (e.g., modulation settings) with SUs. This approach can be practical for an operator-aided CR network, where all the SUs and PUs are serviced by the same operator.

Recently, the generalized-gamma (GG) fading channel, which covers a large number of wellknown fading channel models, has become popular, and many researchers have been studying the wireless system performance over this type of channel [5-10]. In [5], in 2005, Aalo et al. studied the bit error rate (BER) of modulation schemes in GG channels. Then, in 2009, Malhotra et al. investigated the receiver performance over GG fading channels and derived the analytical BER results for $M$-ary phase-shift keying (M-PSK) and $M$-ary quadrature amplitude modulation (M-QAM) schemes using moment-generating function (MGF) approach and Padé approximation technique [6]. Later, in 2011, Gazi derived the upper bounds for outage probability and MGF [7]. A cognitive relay network was studied in [8] and the outage probability was derived for different tolerable interference levels. Additionally, in [9], outage probability was studied for a dual-hop decode-and-forward relay over a GG fading channel. In 2015, 
physical layer security was studied over a GG fading channel in [10]; further, the channels between the receiver and an eavesdropper, as well as that between the receiver and transmitter, were characterized with different GG fading parameters. The outage probability of a cognitive relay network under interference constraints was studied in [11, 12]. A multiple-input multiple-output cognitive relay network, which uses an underlay approach, was considered in [11], and the outage/error performance analysis was presented by means of closed-form expressions over Rayleigh fading channels. The same scenario is also analyzed over composite asymmetric multipath/shadowing fading channels in [12]. Moreover, the outage performance of cognitive DF relay networks for nonidentical independent Rayleigh fading channels, nonidentical interference power limits of PUs, and nonidentical maximum transmission power limits of SUs have been analyzed in $[13,14,15]$. A similar work is also presented in [16] involving cognitive AF relay networks.

In this paper, it is assumed that the CR network employs the underlay approach; therefore, the transmit power of SUs is limited to guarantee that SU transmission is not harmful to PU transmission. The channels between the SU transmitter and SU receiver, as well as those between the SU transmitter and PU receiver, are modeled with GG fading distribution. Simulations are performed for various values of the fading parameters of the GG fading distribution. The contribution of this study is the investigation of the effect of limited SU power on the BER performance as well as on ergodic capacity over a GG fading channel, which is not represented in earlier studies.

This paper is organized as follows. Section 2 describes the system model under consideration. Section 3 presents the analysis of outage probability, ergodic capacity, and BER performance. Section 4 provides the simulation results. Finally, the paper concludes with Section 5 .

\section{System Model}

The $\mathrm{CR}$ network model under consideration is shown in Figure 1. While the transmitter of the $\mathrm{SU}$ is denoted by $S U_{T X}$, the PU

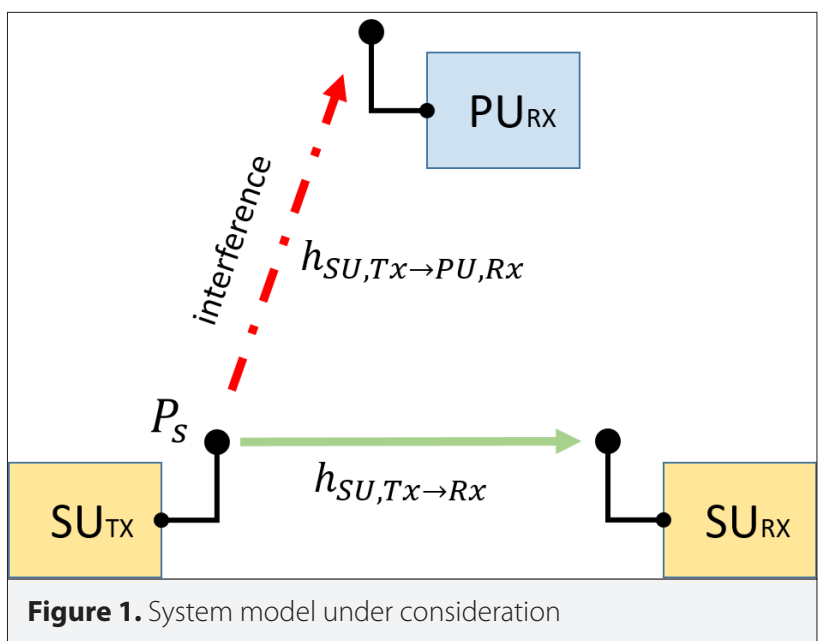

and SU receivers are denoted by $P U_{R X}$ and $S U_{R X}$, respectively. Each node has a single antenna. The transmit power of the SU, $P_{s^{\prime}}$ is determined by $P_{s}=\min \left(\frac{Q_{p}}{E\left\{\left|\mathrm{~h}_{\text {sux } x-\operatorname{sux}}\right|^{1}\right\}}, P_{\max }\right)$, where $Q_{p}$ and $P_{\max }$ denote the maximum tolerable interference level and maximum transmit power level, respectively. $\mathrm{h}_{\mathrm{SU}, \mathrm{TX} \rightarrow P \mathrm{R}, \mathrm{Rx}}$ represents the fading channel information between $S U_{T X}$ and $P U_{R X}$. The transmission channel is modeled as a GG random variable. The GG distribution is given by $[7,10]$

$$
f_{R}(r)=\frac{\alpha c^{c} r^{\alpha c-1}}{\Gamma(c) \bar{r}^{\alpha c}} \exp \left(-c\left(\frac{r}{\bar{r}}\right)^{\alpha}\right), \alpha>0, c>0
$$

where a denotes the fading parameter; $c$ and $\bar{r}$ represent the normalized variance and a-root mean value of the channel envelope $R$, respectively. Finally, $\Gamma(c)=\int^{\infty} t^{c-1} e^{-t} d t$ is the wellknown gamma function. A GG fading channel can be transformed into other distributions through the use of different fading parameters and variance values. Table 1 lists the corresponding distributions of a GG fading channel with different a and $c$ values.

Because the fading channel is modeled as GG, the probability density function and cumulative distribution function (CDF) of the signal-to-noise ratio (SNR) is expressed as [10]

$$
\begin{aligned}
& f(\gamma)=\frac{\alpha c^{c} \gamma^{\alpha c / 2-1}}{2(\bar{\gamma})^{\alpha c / 2} \Gamma(c)} \exp \left(-c\left(\frac{\gamma}{\bar{\gamma}}\right)^{\alpha / 2}\right), \\
& F(\gamma)=\frac{\Upsilon\left(c, c\left(\frac{\gamma}{\bar{\gamma}}\right)^{\alpha / 2}\right)}{\Gamma(c)}
\end{aligned}
$$

where $\Upsilon(\alpha, x)=\int_{0}^{x} e^{-t} t^{\alpha-1} d t$ is the lower incomplete gamma function, as defined in [17, Eq.(8.350/1)]. SNR is expressed as

$$
\gamma=\min \left(\frac{Q_{p}}{E\left\{\left|\mathrm{~h}_{\text {su.TX } \rightarrow \text { PU.RX }}\right|^{2}\right\}}, P_{\max }\right)\left|\mathrm{h}_{\mathrm{su}, \mathrm{TX}-\mathrm{SU}, \mathrm{RX}}\right|^{2},
$$

where $\mathrm{h}_{\mathrm{su}, \mathrm{TX} \rightarrow S \mathrm{U}, \mathrm{Rx}}$ denotes the fading channel information between $S U_{T X}$ and $S U_{R X}$. Let $x_{s}$ denote the transmitted symbol of the SU. The received signal at the SU receiver can be expressed as

$$
y=P_{s} \mathrm{~h}_{\mathrm{su}, \mathrm{TX} \rightarrow S \mathrm{U}, \mathrm{RX}} x_{s}+n_{s},
$$

where $n_{s}$ denotes the complex additive white Gaussian noise (AWGN) with zero mean and unit variance $C N(0, N o / 2)$. Here the interference of PU on the SU is neglected.

Table 1. Various $a$ and $c$ values of a GG fading channel

\begin{tabular}{lll}
\hline $\boldsymbol{a}^{\mathbf{1}}$ & $\boldsymbol{c}^{\mathbf{2}}$ & Corresponding Distributions \\
\hline 2 & 1 & Rayleigh \\
\hline 2 & $c$ & Nakagami-m \\
\hline$a / 2$ & 1 & Weibull \\
\hline$a^{1}$ Denotes a GG fading parameter \\
$c^{2}$ Represents the normalized variance of a GG channel envelope
\end{tabular}




\section{Performance Analysis}

In this paper, the outage probability, ergodic capacity, and BER for the SU are analyzed. These performance metrics are derived for the considered scenario in GG channels.

\section{Outage Probability}

One of the important performance criteria is the outage probability. It can be easily obtained from

$$
P_{\text {out }}=p\left(\gamma \leq \gamma_{\text {thr }}\right)=\int_{0}^{\gamma_{t h r}} f_{\gamma}(\gamma) d \gamma,
$$

where $\gamma_{\text {thr }}$ denotes the predefined threshold value. In GG fading channels, the outage probability can be calculated by using the CDF of SNR, which is given in Eq.(3). In [10], the lower incomplete gamma function is given in the form of Meijer's G-function [17, Eq.(9.301)] as

$\Upsilon\left(c, c\left(\frac{\gamma}{\bar{\gamma}}\right)^{\alpha / 2}\right)=\frac{\Gamma(c+1)}{\Gamma(c) c}\left(c\left(\frac{\gamma}{\bar{\gamma}}\right)^{\alpha / 2}\right)^{c} G_{1,2}^{1,[}\left[c\left(\frac{\gamma}{\bar{\gamma}}\right)^{\alpha / 2} \begin{array}{l}1-c \\ 0, c\end{array}\right]$

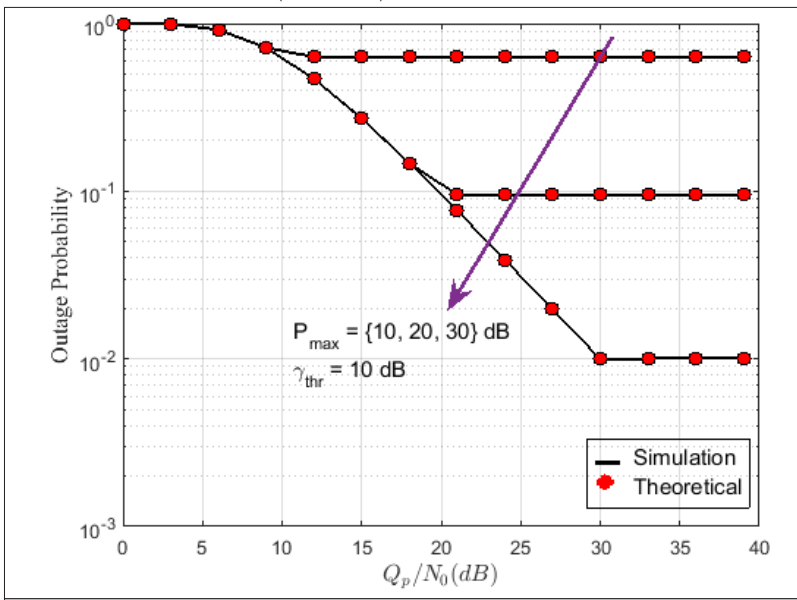

Figure 2. Outage probability vs. $\bar{Q}_{p}$ for different $P_{\max }$ values over a Rayleigh fading channel $\left(a_{s}=2, a_{d}=2 c=1\right)$

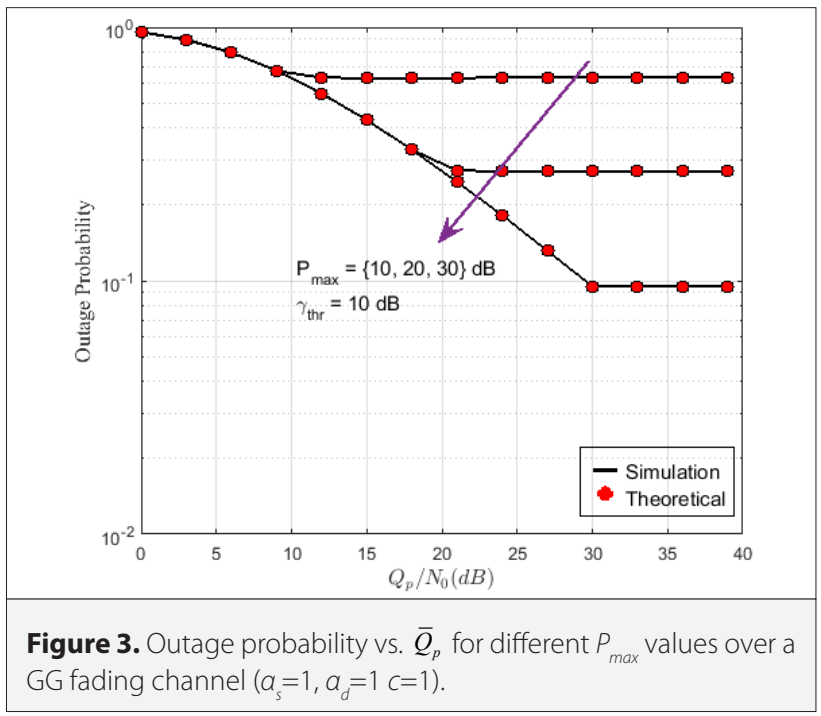

By using Eq.(7), the outage probability can be calculated for GG fading channels.

\section{Ergodic Capacity}

The well-known capacity equation is given in Eq.(8):

$$
C=\mathrm{E}\left[\log _{2}(1+\gamma)\right],
$$

where $\gamma$ represents the SNR value of the SU, which depends on the random fading channel. By using Jensen's inequality, the upper bound of Eq.(8) can be expressed as

$C \leq \log _{2}(1+\bar{\gamma})$,

where $\bar{\gamma}$ represents the average SNR. By using Meijer's G-function representation of $F_{\gamma}(\gamma)$ in Eq.(7), $\bar{\gamma}$ can be calculated as

$\bar{\gamma}=\int_{0}^{\infty}(1-F(\gamma)) d \gamma=\int_{0}^{\infty}\left(1-\Upsilon\left(c, c\left(\frac{\gamma}{\bar{\gamma}}\right)^{\alpha / 2}\right)\right) d \gamma$.

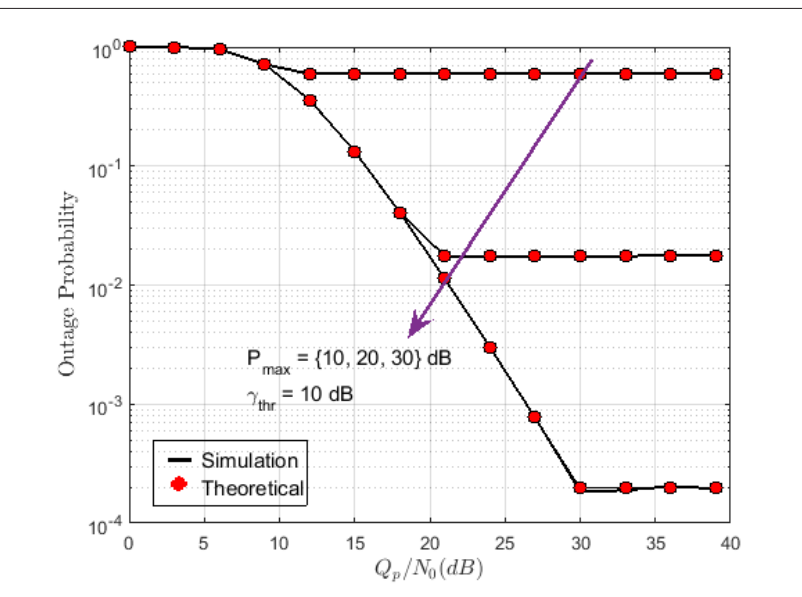

Figure 4. Outage probability vs. $\bar{Q}_{p}$ for different $P_{\max }$ values over a Nakagami fading channel $\left(a_{s}=2, a_{d}=2 c=2\right)$

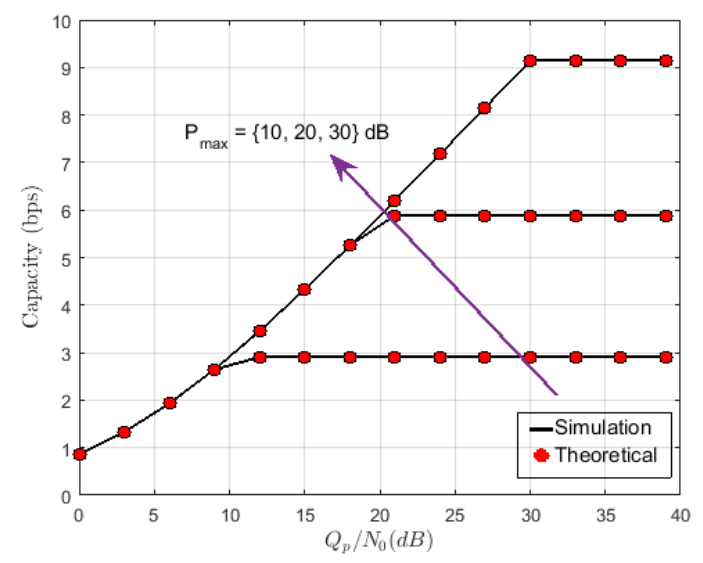

Figure 5. Capacity variation vs. $\bar{Q}_{p}$ for different $P_{\text {max }}$ values over a Rayleigh fading channel $\left(a_{s}=2, a_{d}=2, c=1\right)$ 
When the fading parameters are chosen as $a_{s}=2, a_{d}=2$, and $c=1$, the GG fading channel turns into a Rayleigh channel, and therefore, the capacity can be calculated as

$$
C=\frac{1}{\ln 2} \int_{x}^{\infty} \frac{e^{-t}}{t} d t
$$

The theoretical curve is only given for the Rayleigh fading case $\left(a_{s}=2, a_{d}=2\right.$, and $\left.c=1\right)$. For the other cases, the capacity results are obtained with Monte Carlo simulations.

\section{Bit Error Rate Analysis}

In the system model under consideration, the average BER can be calculated by averaging over the fading channel:

$\bar{P}_{E}=\int_{0}^{\infty} P_{E}(\gamma) f_{\gamma}(\gamma) \mathrm{d} \gamma$,

where $P_{E}(\gamma)=\frac{\Gamma(b, a \gamma)}{2 \Gamma(b)}$ denotes the conditional BER in the AWGN channel [18, Eq.(8.100)]. Further, $a$ and $b$ values depend on
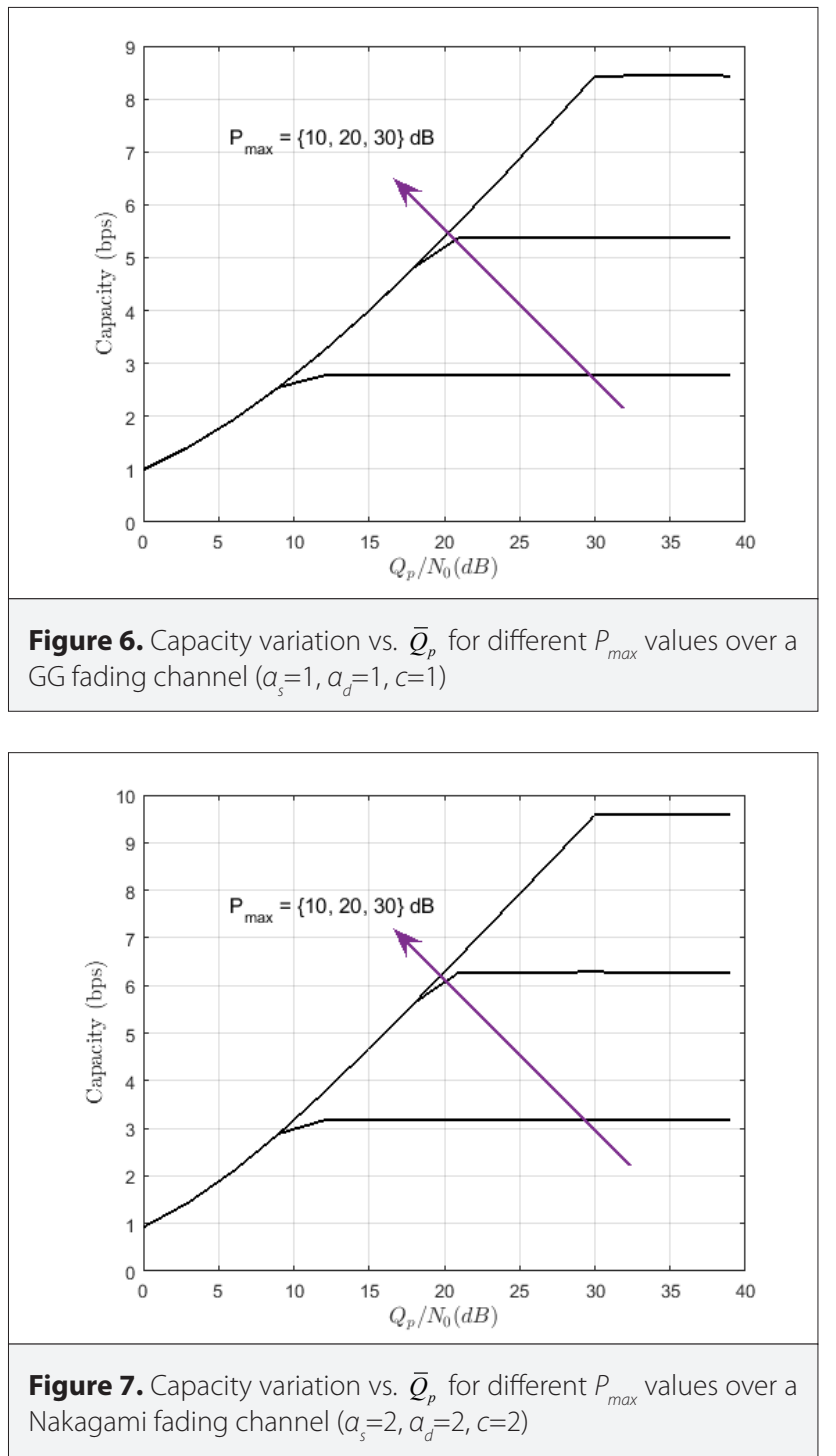

the modulation type and demodulation process. For BPSK modulation and coherent detection, $a=1$ and $b=0.5$. The average BER is obtained from [5]:

$$
\bar{P}_{E}=A(\mathrm{k}, \mathrm{l}) \mathrm{G}_{2 l, k+l}^{k, 2 l}\left[\left(\frac{l \beta}{k^{k / l} a \bar{\gamma}}\right)^{l} \mid \begin{array}{c}
\alpha_{1}, \mathrm{~K}, a_{2 l} \\
\beta_{1}, \mathrm{~K}, \beta_{k+l}
\end{array}\right],
$$

where

$\alpha_{n}=\left\{\begin{array}{cc}1-\frac{n+b-1}{l} & n=1,2, \mathrm{~K}, l \\ 1-\frac{n-l-1}{l} & n=l+1, l+2, \mathrm{~K}, 2 l\end{array}\right.$,

and

$\beta_{n}=\left\{\begin{array}{c}\frac{n+c-1}{k} \quad n=1,2, \mathrm{~K}, k \\ 1-\frac{n-k}{l} \quad n=k+1, k+2, \mathrm{~K}, \mathrm{k}+l\end{array}\right.$

Moreover, $\beta=\frac{\Gamma(c+2 / \alpha)}{\Gamma(c)}$ and $\alpha / 2=l / k$.

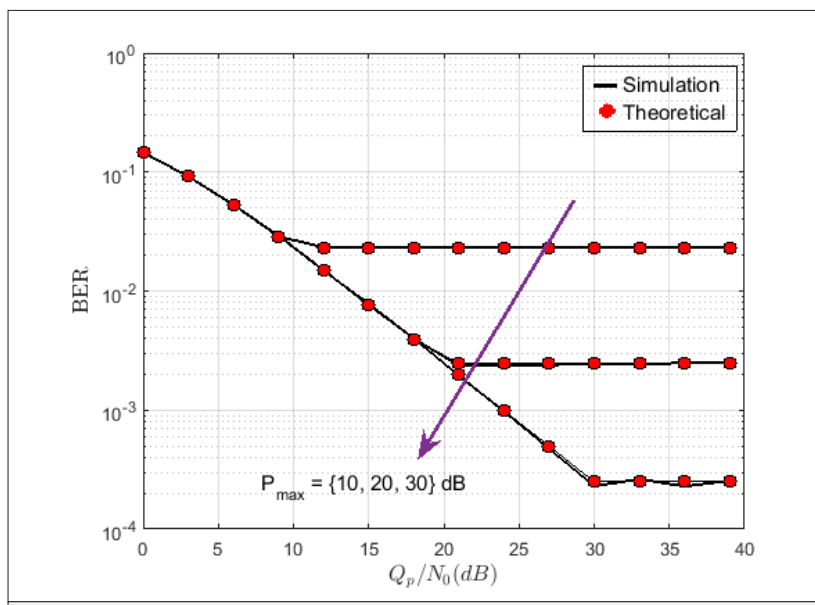

Figure 8. Bit error probability vs. $\bar{Q}_{p}$ for different $P_{\max }$ values over a Rayleigh fading channel $\left(a_{s}=2, a_{d}=2, c=1\right)$

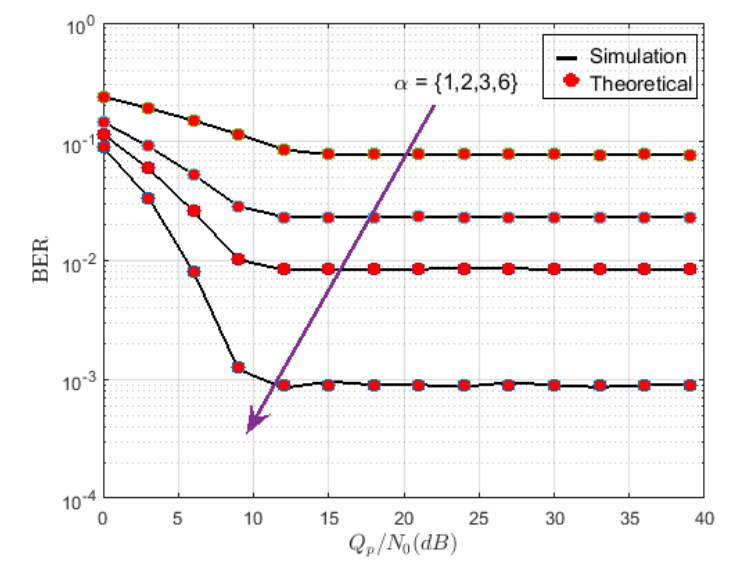

Figure 9. Bit error probability for various a fading parameters $\left(P_{\max }=10 \mathrm{~dB}, \mathrm{C}=1\right)$ 


\section{Simulation Results}

In this section, the outage probability, capacity, and BER performance of the SU in the underlay CR network are investigated. In all the simulations, it is assumed that the SU's modulation type is BPSK, $N_{0}=1$, and the tolerable interference-to-noise ratio $\bar{Q}_{p}=Q_{p} / N_{0}$ changes from 0 to $40 \mathrm{~dB}$. However, the SU's power is limited to $P_{\max }$ to avoid causing interference to the PU. In the simulations, three different $P_{\max }$ values are considered, namely, $P_{\text {max }}=\{10,20,30\} \mathrm{dB}$. All the simulations are performed by using Monte Carlo with $10^{6}$ iterations (except for the last one, which is obtained with $10^{7}$ iterations) using MATLAB (MathWorks, Natick, Massachusetts, U.S.A). The outage probability, capacity, and BER performance are investigated for various $a$ values ( $a=1,2,3$, and 6$)$. To simplify the notation, the $a$ values of $\mathrm{h}_{\mathrm{SU} . \mathrm{TX} \rightarrow P \mathrm{U} . \mathrm{RX}}$ and $\mathrm{h}_{\mathrm{SU}, \mathrm{TX} \rightarrow S \mathrm{U}, \mathrm{RX}}$ are represented as $a_{d}$ and $a_{s}\left(a_{d^{\prime}} a_{s}\right)$, respectively.

Outage probability, capacity, and BER performance are obtained for different $\bar{Q}_{p}$ and $P_{\max }$ values. First, theoretical and
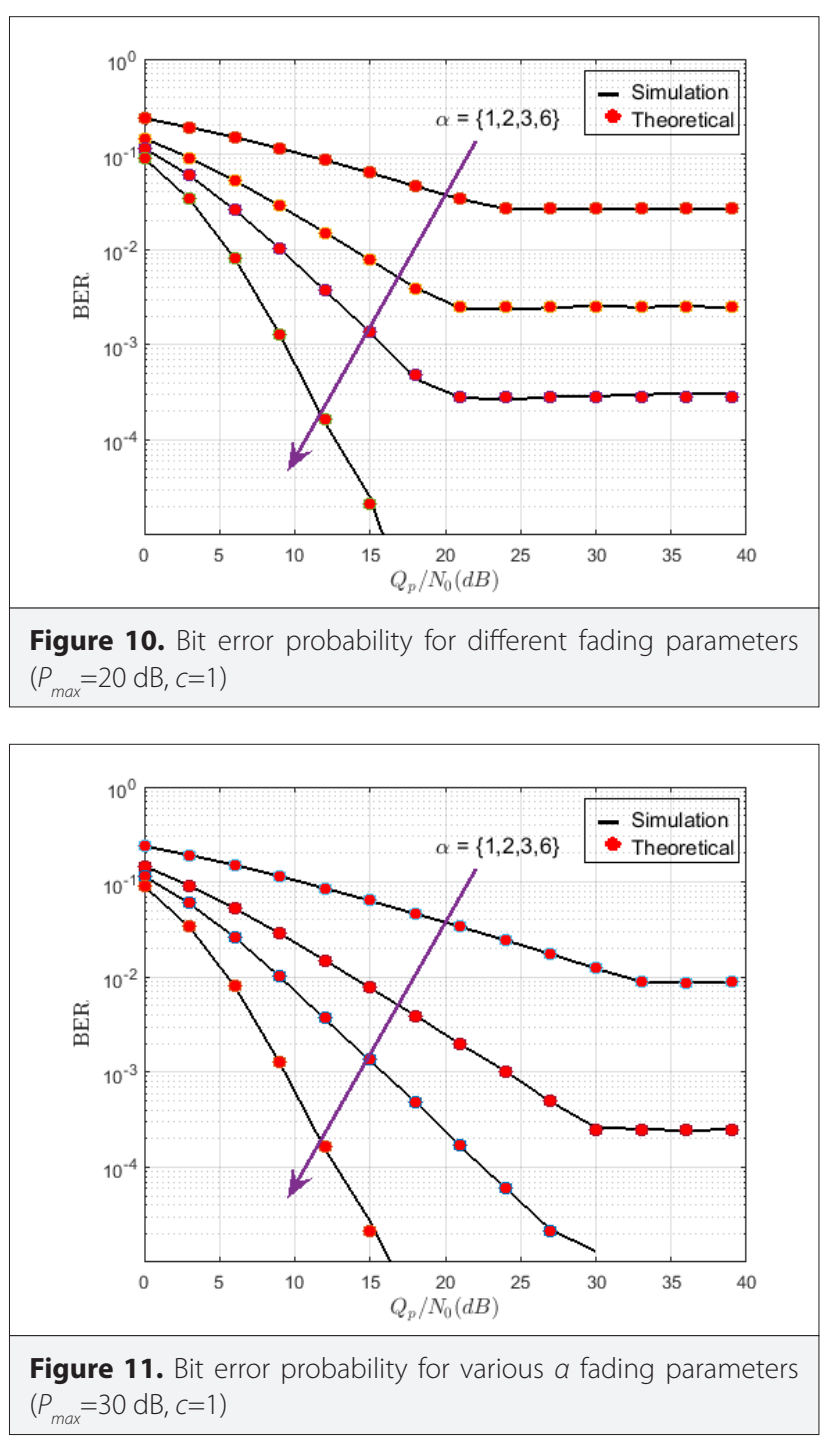

simulation outage probability values are shown in Figure 2, Figure 3, and Figure 4 for various channel fading parameters. When the fading channel parameters are chosen as $a_{s}=2, a_{d}=2$, and $c=1$ (Figure 2), the GG fading channel turns into a Rayleigh fading channel. In addition, Figure 4 shows the outage probability over a Nakagami-m fading channel $\left(a_{s}=2, a_{d}=2\right.$, and $c=2$ ). In all these figures, the threshold value $\gamma_{\text {thr }}$ is chosen as $10 \mathrm{~dB}$. It is clear that the theoretical results match very well with the simulation curves. As the transmission power increases, the outage probability improves. The worst outage probability is obtained in Figure 3, because the channel fading effect is severe as compared to those of the analyzed channels shown in Figure 2 and Figure 4. The lowest outage probability value is obtained as almost $10^{-2}$ for the Rayleigh fading channel, while this value is close to $10^{-4}$ for the Nakagami fading channel when $P_{\max }=30 \mathrm{~dB}$.

Figure 5 shows the capacity variations for different $P_{\max }$ values in a Rayleigh fading channel $\left(a_{s}=2, a_{d}=2\right.$, and $\left.c=1\right)$. As shown in the figure, for all the $P_{\max }$ values, the capacity first increases as $\bar{Q}_{p}$ increases and then it saturates when it reaches the predefined threshold value. The theoretical capacity values perfectly match with the simulation results for all the $P_{\max }$ values.

Figure 6 and Figure 7 show the capacity performance of SUs in different fading channels. Evidently, when the c value is 2 instead of 1, higher capacity values can be reached. Further, when all the fading parameters are 1 , the worst channel is obtained. For example, when $P_{\max }$ is selected as $30 \mathrm{~dB}$, while the capacity value equals 7 bps at $25 \mathrm{~dB}$ (Figure 6), the capacity equals 8 bps at the same $\bar{Q}_{p}$ value (25 dB) (Figure 7).

Finally, the theoretical and simulation BER results are given for different $a$ fading channel parameters and various maximum limited transmit power values. In all the simulation setups, the simulated BER results perfectly match with the theoretical results. First, the channel parameters are chosen as $a_{s}=2, a_{d}=2$, and $c=1$ (Rayleigh fading), and the BER results are obtained both via simulation and analysis. These results are shown in

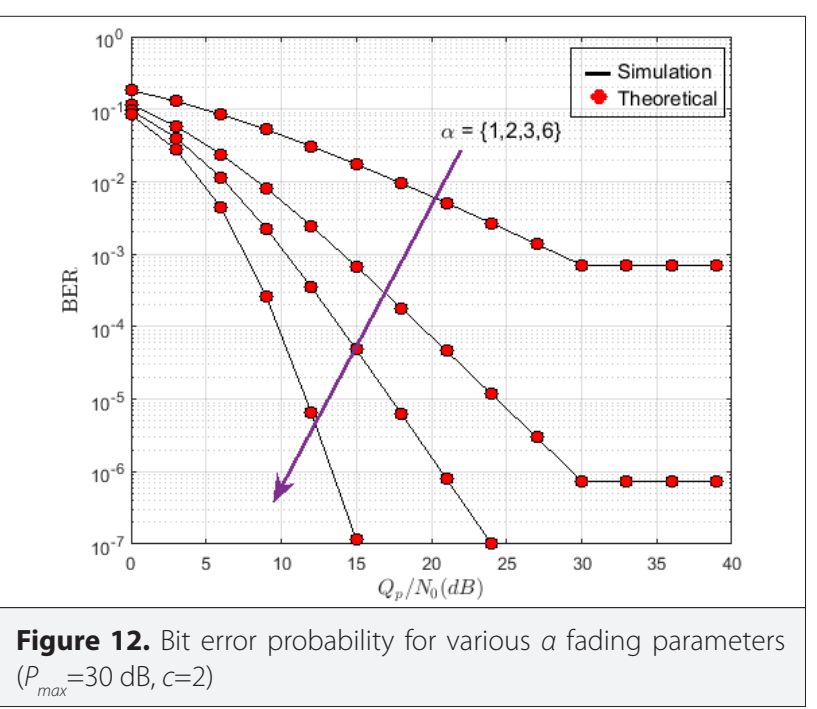


Figure 8. As expected, for $P_{\max }=30 \mathrm{~dB}$, the BER performance remarkably improves. All the BER curves perform almost the same until $\bar{Q}_{p}=10 \mathrm{~dB}$; then, the smallest $P_{\max }$ value $\left(P_{\max }=10 \mathrm{~dB}\right)$ is saturated. At $\bar{Q}_{p}=18 d B$, the BER performance for $P_{\max }=20 \mathrm{~dB}$ is saturated. The BER value at this saturation point is 0.0024 . For $P_{\max }=30 \mathrm{~dB}$, the curve outperforms $P_{\max }=10$ and $20 \mathrm{~dB}$. In Figures $9-11$, it is assumed that $c=1$ and $a$ values are set to $a=\{1$, $2,3,6\}$ to evaluate the BER performance with various limited SU transmit power $\left(P_{\max }\right)$ values. The dominant effect on BER depends on the selection of $P_{\text {max }}$. In Figure 12, $\mathrm{c}$ is set to 2 . Evidently, as $a$ increases, the BER performance significantly improves. To determine the effect of $c$ on the BER performance, Figure 11 and Figure 12 can be compared. Evidently, while the SNR gap equals $12 \mathrm{~dB}$ at $\mathrm{BER}=10^{-2}$ when $a=1$, this gap equals $9 \mathrm{~dB}$ at $B E R=10^{-3}$. The SNR gap decreases with increasing a.

\section{Conclusion}

In this paper, the outage probability, ergodic capacity, and BER performance of SUs over a GG fading channel in the underlay CR system are investigated. The closed-form expressions are also given, and the obtained results closely match with those obtained from the simulations. Because of the limited transmit power of SUs, the performance indicators, namely, outage probability, BER, and ergodic capacity, saturate with the $P_{\text {max }}$ value. When this limited power is increased, all the performance metrics improve.

Peer-review: Externally peer-reviewed.

Conflict of Interest: The author have no conflicts of interest to declare.

Financial Disclosure: The author declared that the study has received no financial support.

\section{References}

1. J. III, Mitola, Cognitive radio: An integrated agent architecture for software defined radio. Ph.D. thesis, KTH Royal Institute of Technology, 2000.

2. J. III, Mitola, Cognitive radio for flexible mobile multimedia communication. In: Proceedings of IEEE International Workshop on Mobile Multimedia Communications (MoMuC), San Diego, CA, 1999.

3. S. Haykin, "Cognitive radio: Brain-empowered wireless communications", IEEE Journal on Selected Areas in Comm., vol. 23, no. 2, pp. 201-220, 2005. [CrossRef]

4. I. F. Akyildiz, W. Y. Lee, M. C. Vuran, S. Mohanty, "Next generation/ dynamic spectrum access/cognitive radio wireless networks: A survey", Computer Networks, vol. 50, no. 3, pp. 2127-2159, 2006. [CrossRef]
5. V. A. Aalo, T. Piboongungon, C. D. Iskander, "Bit-error rate of binary digital modulation schemes in generalized gamma fading channels", IEEE Communications Letters, vol. 9, no. 2, pp.139-141, 2005. [CrossRef]

6. J. Malhotra, A.K. Sharma, R.S. Kaler. "On the performance analysis of wireless receiver using generalized-gamma fading model", Annals of Telecommunications-Annales des Télécommunications, vol. 64, no. 1-2, pp. 147-153, 2009. [CrossRef]

7. O. Gazi, "Bounds for generalized gamma distributed fading channels", IEEE Communications Letters, vol. 15, no. 12, pp. 1347-1349, 2011. [CrossRef]

8. P.S. Bithas, G.P. Efthymoglou, D.S. Kalivas, "Outage probability of cognitive relay networks over generalized fading channels with interference constraints", Procedia Computer Science, vol. 40 pp. 84-91, 2014. [CrossRef]

9. N. Kapucu, M. Bilim, I. Develi, “Outage probability analysis of dual-hop decode-and-forward relaying over mixed Rayleigh and generalized Gamma fading channels", Wireless Personal Communications, vol. 71, no. 2, pp.947-954, 2013. [CrossRef]

10. H. Lei, C. Gao, Y. Guo, G. Pan, G. "On physical layer security over generalized Gamma fading channels", IEEE Communications Letters, vol. 19, no. 7, pp. 1257-1260, 2015. [CrossRef]

11. E. Erdogan, A. Afana, S. Ikki, H. Yanikomeroglu, "Antenna selection in MIMO cognitive AF relay networks with mutual interference and limited feedback", IEEE Communications Letters, vol. 21, no. 5, pp. 1111-1114, 2017. [CrossRef]

12. E. Erdogan, "Cognitive AF Relay Networks over Asymmetric Shadowing/Fading Channels in the Presence of Low-Rate Feedback", Electrica, vol. 18, no. 2, pp. 172-176, 2018. [CrossRef]

13. Z. Gao, D. Chen, N. Yao, Z. Lu, B. Chen, G. Tan, " Outage Probability of Cognitive Selective DF Relay Networks with Multiple Primary Nodes and Heterogenous Non-Identical Constraints", Wireless Personal Communications, vol. 92, no. 3, pp. 969-992, 2017. [CrossRef]

14. Z. Gao, D. Chen, B. Chen, Z. Lu, N. Yao, "Outage probability equivalency of three typical relay selection schemes for selective DF relay networks with selection combining", Wireless Personal Communications, vol. 85, no. 3, pp.1205-1215, 2015. [CrossRef]

15. Z. Gao, K. Zhang, D. Chen, W. Zhang, Y. Li, "Outage performance of cognitive DF relay networks with nonidentical Rayleigh fading channels and maximal ratio combining", AEU-International Journal of Electronics and Communications, vol. 69, no. 1, pp. 141-150, 2015. [CrossRef]

16. Z. Gao, D. Chen, K. Zhang, W. Zhang, Y. Li, "Outage performance of cognitive AF relay networks with direct link and heterogeneous non-identical constraints", Wireless Communications and Mobile Computing, vol. 16, no. 6, pp.669-681, 2016. [CrossRef]

17. I. S. Gradshteyn, I.M. Ryzhik, "Table of integrals, series and products," 7th ed. New York: Academic Press, 2007.

18. M. K. Simon, M. S. Alouini, "Digital Communication over Fading Channels - A Unified Approach to Performance Analysis", Wiley Interscience, 2000. [CrossRef] 
Sultan Aldırmaz Çolak received the B.S degree in Electronics and Communications Engineering from Kocaeli University, Kocaeli 2004 and the M.S. and PhD degrees in Yildiz Technical University (YTU), Istanbul 2006 and 2012, respectively. She was a visiting research scholar in the Department of Electrical and Computer Engineering of University of South Florida for the spring and summer of 2009. She is currently an Assistant Professor in the Electronics and Communications Engineering Department of Kocaeli University, Kocaeli, Turkey. Her research interests are in time-frequency signal processing, and communications theory. 